\title{
Cyclin-dependent kinase 4/6 inhibitor in combination with endocrine therapy versus endocrine therapy only for advanced breast cancer: a systematic review and meta-analysis
}

\author{
Zhi-Hong Xu ${ }^{1 \#}$, Heng Zhang ${ }^{1 \#}$, Deng-Hui Wei ${ }^{1}$, Li-Li Xie ${ }^{1}$, Chun-Sen Xu ${ }^{2}$ \\ ${ }^{1}$ Fujian Medical University, Fuzhou 350004, China; ${ }^{2}$ Department of Breast Surgery, Fujian Medical University Union Hospital, Fuzhou 350001, \\ China \\ Contributions: (I) Conception and design: ZH Xu, H Zhang; (II) Administrative support: DH Wei, LL Xie; (III) Provision of study materials or \\ patients: ZH Xu, H Zhang, CS Xu; (IV) Collection and assembly of data: DH Wei, LL Xie; (V) Data analysis and interpretation: ZH Xu, DH Wei, \\ LL Xie; (VI) Manuscript writing: All authors; (VII) Final approval of manuscript: All authors. \\ \#These authors contributed equally to this work. \\ Correspondence to: Chun-Sen Xu, MD. Department of Breast Surgery, Fujian Medical University Union Hospital, No. 29 of Xinquan Road, Gulou \\ District, Fuzhou 350001, China. Emil: xuchunsen909@163.com.
}

\begin{abstract}
Background: The resistance to endocrine therapy poses a significant challenge to the management of advanced breast cancer with hormone receptor (HR) positive and human epidermal growth factor receptor 2 (Her-2) negative. The purpose of this study was to further examine the efficacy and safety of cyclindependent kinase 4/6 inhibitors (CDK4/6Is) in combination with endocrine therapy as a recovery treatment for advanced breast cancer patients.
\end{abstract}

Methods: The risk of bias for each included study was assessed using the Cochrane Risk of Bias Tool. The Cochrane $Q$ value, combined with the $\mathrm{I}^{2}$ statistics, were selected to be tested for heterogeneity across the studies. The generic inverse variance was used to pool the hazard ratio and $95 \%$ CI of progression-free survival (PFS) and overall survival (OS), while pooled RRs and 95\% CI were conducted using the MantelHaenszel to appraise the overall response rate (ORR), clinical benefit rate (CBR), and any adverse effects.

Results: Eight random clinical trials were finally identified. The analysis showed that the duration of PFS was significantly longer in the CDK4/6Is group than in the control group (hazard ratio, 0.55; 95\% CI, 0.510.60; $\mathrm{P}<0.00001$ ), and treatment with CDK4/6Is-endocrine therapy resulted in longer OS than treatment with endocrine therapy only (hazard ratio, 0.79; 95\% CI, 0.66-0.96; $\mathrm{P}=0.001$ ). As for any adverse events, the analysis showed a remarkable rise in bone marrow suppression, especially neutropenia and leukopenia (respectively, RR =32.04; 95\% CI, 17.14-59.90, RR =30.65; 95\% CI, 16.51-56.91), but not in gastrointestinal toxicity.

Conclusions: Highly selective CDK4/6Is were well tolerated, effective drugs in advanced breast cancer patients with HR-positive and Her-2 negative.

Keywords: Breast cancer; cyclin-dependent kinase; endocrine therapy; meta-analysis

Submitted Jun 28, 2019. Accepted for publication Oct 10, 2019.

doi: $10.21037 /$ tcr.2019.11.46

View this article at: http://dx.doi.org/10.21037/tcr.2019.11.46

\section{Introduction}

Breast cancer is the most common malignant tumor threatening the health of women worldwide. The incidence and mortality of breast cancer around the world shows an upward tendency year by year (1). In America, an annual report showed that 246,660 women were diagnosed with breast cancer in 2016 (2), with 40,450 women dying from the advanced or metastatic disease. 
Heterogeneity is a major feature of breast cancer, including the four distinct molecular subtypes (3). Luminal-types make up three-quarters of breast cancer (4). Consequently, endocrine therapy makes up a significant component of the comprehensive treatment. The use of endocrine therapy as a recovery treatment of HRpositive, Her-2 negative advanced breast cancer patients is recommended in the clinical guidelines, especially for the patients without significant visceral disease or rapid tumor progression. Despite the fact that endocrine therapy has made great progress in the treatment of breast cancer, some patients do not respond to endocrine therapy due to intrinsic or acquired resistance.

Another hallmark of breast cancer is sustained proliferation due to the dysregulation of the cell-cycle. Normal cell division is under a very specific program, and retinoblastoma $(\mathrm{Rb})$ protein plays a switching role in the cell-cycle. The extracellular stimulation of pro-mitotic signals can increase the expression of cyclin D1, which can complex with and activate cyclin-dependent kinases 4 and 6 , leading to the phosphorylation of $\mathrm{Rb}$ protein, which finally increases the expression of S-phase-specific genes and initiate G1-to-S phase transition (5). Any mechanism that disturbs the cyclin D1-CDK4/6-Rb pathway may promote cell proliferation, including amplification of the cyclin D1 gene and overexpression of cyclin D1. Simultaneously, preclinical studies have shown that resistance to endocrine therapy is also related to the dysregulation of the cell cycle $(6,7)$. The search for CDK4/6Is targeting the cyclin D1CDK4t/6-Rb pathway in breast cancer is a significant achievement $(5,8)$, and the advent of CDK4/6Is offers a new approach to advanced breast cancer patients.

First generation CDK inhibitors were tested in many clinical trials (9-14), showing limited clinical benefit and an unacceptable toxicity profile, eventually causing them to be discontinued. With the development of the agents, the first-in-class, highly selective inhibitor of cyclindependent kinases 4 and 6, palbociclib, was developed by Pfizer in 2012. Since the results of a random, phase II study (PALOMA-1/TRIO-18) (15) verified the safety and clinical benefit of Palbociclib, the FDA approved it. Meanwhile, it was observed in other clinical trials that PFS was significantly improved in the group treated with ribociclib/abemaciclib and endocrine therapy. Although the safety and clinical benefits of CDK4/6Is plus endocrine were underpinned by previous clinical trials, there was no formal head to head comparison between these drugs. Recently, Ramos-Esquivel et al. (16) conducted a meta- analysis showing an obviously curative effect of CDK4/6Is plus aromatase inhibitor compared with an aromatase inhibitor only. However, only three clinical trials were eligible for this meta-analysis, and the clinical trials were restricted to phase III. In addition, data of the hazard ratio in this meta-analysis were different from the original data. As more and more clinical results came out, it was necessary to perform a meta-analysis to provide more significant evidence for the efficacy and safety of CDK4/6Is in combination with endocrine therapy as salvage treatment.

\section{Methods}

\section{Literature search strategy}

We systematically searched PubMed, Embase, Cochrane Library from inception until December 12, 2018, and conducted an electronic search of the main international congress abstracts. The search terms were listed as the following: "breast cancer", "CDK4/6 inhibitor", "Palbociclib", "Ribociclib", "Abemaciclib", "Flavopiridol", "R-Roscovitine", "endocrine therapy", "Tamoxifen", "Letrozole", "Anastrozole", "Exemestane" and "Fulvestrant".

\section{Inclusion criteria}

We screened out the phase II or III random clinical trials that assessed the efficacy and safety of CDK4/6Is plus endocrine therapy compared with endocrine therapy only, recruiting advanced breast cancer patients with HR-positive and Her-2 negative.

\section{Data extraction}

We searched the four past and recent annual meetings to identify all the eligible articles and abstracts using the strategy mentioned above. Two investigators extracted the following information from each study: the surname of the first author, year of publication, style of the trials, treatment arms, patients, the hazard ratio for PFS and $95 \% \mathrm{CI}$ (Table 1), the overall response and clinical benefit rate (CBR). The divergent opinion was judged by the third investigator.

\section{Quality assessment}

We assessed the risk of bias for each included study using the Cochrane Risk of Bias Tool, and we judged each entry 
Table 1 The characteristics of the eight included studies in the analysis

\begin{tabular}{|c|c|c|c|c|c|c|c|c|}
\hline Study & Author & Year & Style & Treatment regiment & Patients & $\mathrm{HR}(95 \% \mathrm{Cl})$ & $\mathrm{InHR}$ & SE \\
\hline PALOMA-3 & Nicholas C. Turner & 2016 & $\begin{array}{c}\text { Phase } 3 \text { double-blind } \\
\text { randomized }\end{array}$ & $\mathrm{P}+\mathrm{F}$ vs. $\mathrm{F}$ & 521 & $0.46(0.32-0.59)$ & -0.777 & 0.156 \\
\hline MONARCH-2 & George W. Sledge & 2017 & $\begin{array}{c}\text { Phase } 3 \text { double-blind } \\
\text { randomized }\end{array}$ & $A+F$ vs. $F$ & 669 & $0.553(0.449-0.681)$ & -0.592 & 0.106 \\
\hline MONARCH-3 & Matthew P. Goetz & 2017 & $\begin{array}{c}\text { Phase } 3 \text { double-blind } \\
\text { randomized }\end{array}$ & $\mathrm{A}+\mathrm{Al}$ vs. $\mathrm{Al}$ & 493 & $0.54(0.41-0.72)$ & -0.616 & 0.144 \\
\hline MONALEESA-2 & G. N. Hortobagyi & 2018 & $\begin{array}{c}\text { Phase } 3 \text { double-blind } \\
\text { randomized }\end{array}$ & $\mathrm{R}+\mathrm{L}$ vs. $\mathrm{L}$ & 668 & $0.568(0.457-0.704)$ & -0.566 & 0.116 \\
\hline MONALEESA-7 & Debu Tripathy & 2017 & $\begin{array}{c}\text { Phase } 3 \text { randomized } \\
\text { double-blind }\end{array}$ & $\begin{array}{c}\text { A+T/NSAI vs. } \\
\text { T/NSAI }\end{array}$ & 672 & $0.553(0.441-0.694)$ & -0.592 & 0.116 \\
\hline
\end{tabular}

P, palbociclib; R, ribociclib; A, abemaciclib; L, letrozole; Al, aromatase inhibitor; T, tamoxifen; NSAl, non-steroidal aromatase inhibitor.

with "low risk", "high risk" and "unclear", supported by the characteristic of each study (17). Simultaneously, we used the funnel plot to assess reporting bias to make the analysis more reliable.

\section{Statistical analysis}

We chose the Cochrane $\mathrm{Q}$ value combined with the $\mathrm{I}^{2}$ statistics to test for heterogeneity across studies. In the case of inter-study heterogeneity, we searched for the source of heterogeneity by comparing the inclusion and exclusion criteria of each clinical trial, the difference in intervention measures, the difference in trial design and other aspects, and further explored the source of heterogeneity with subgroup analysis to reduce the heterogeneity across groups and improve the credibility of the combined effect. We used the generic inverse variance to pool the hazard ratio and $95 \%$ CI of PFS and OS, and pooled RRs and 95\% CI were conducted using the Mantel-Haenszel to appraise the ORR, CBR, and adverse effects. The fixed effect model and random effect model were employed on the basis of heterogeneity analysis. All the analyses were performed using RevMan 5.3 analysis software.

\section{Results}

\section{Characteristics of the studies}

A total of 2,169 articles were identified through the three databases and annual meetings, the repeated findings of which brought eight $(15,18-24)$ eligible trials including 4,580 patients after screening the title or abstract, even the full text. The proceeding for the selection of studies is illustrated in Figure 1. All the trials were randomized clinical trials, and there were only one phase II and seven phase III clinical trials. In addition, the blind method was adopted by all the trials except PALOMA-1. The main characteristics of the included studies is shown in Table 1.

\section{Bias}

The details of risk bias are summarized in Figure 2. Of the six main entries, three trials were regarded as low risk. These eight studies were a randomized clinical trial, and five of them detailed how to generate a random distribution sequence. The blind method was adopted in all the trials except PALOMA-1, and the outcomes assessors were masked to the treatment assignment in six of these trials. 


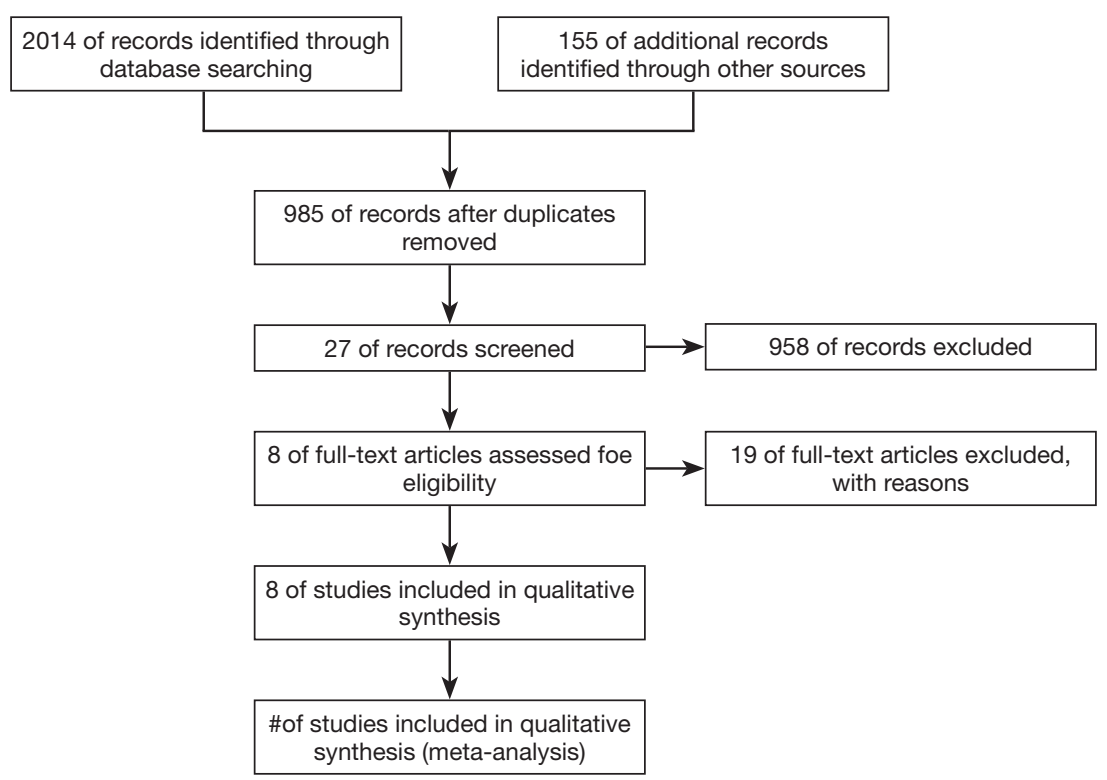

Figure 1 Study flow diagram.

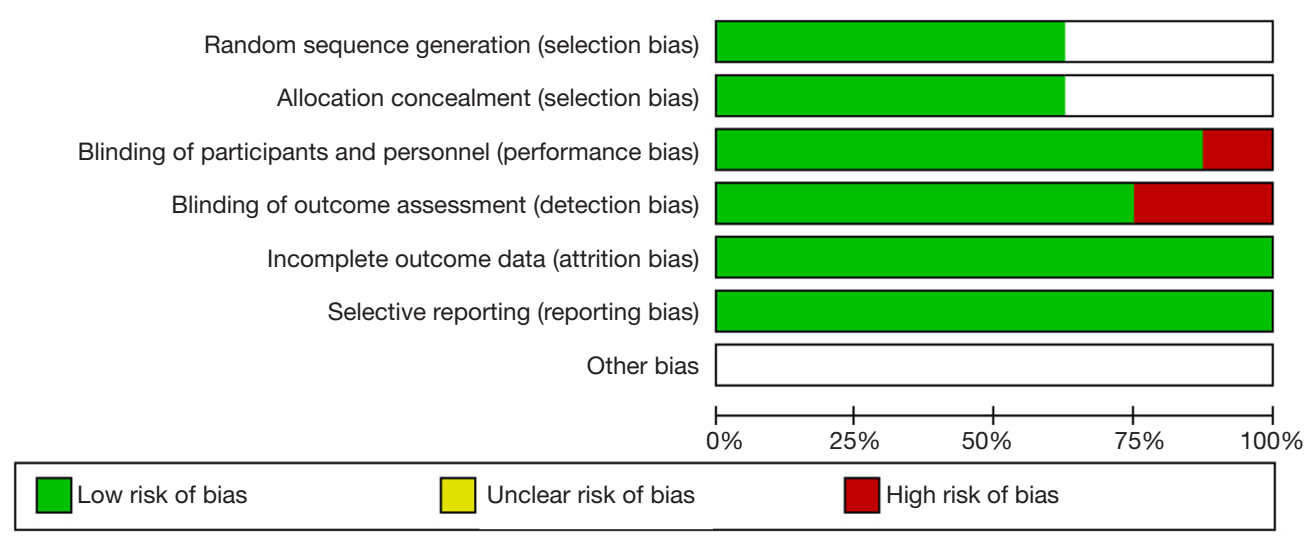

Figure 2 Risk of bias graph.

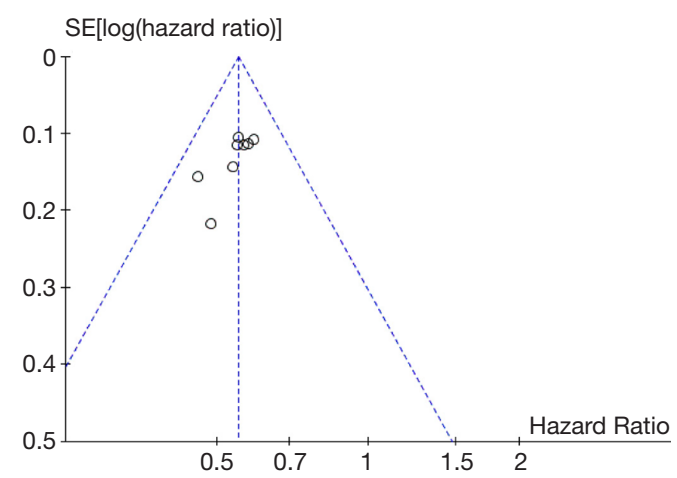

Figure 3 Funnel plot of comparison.
All clinical trials were conducted to follow the research plan and reported all the intended outcome indicators. Detailed data could be found in all the trials. Overall, all trials were at a low risk of bias which ensured the reliability of the results. In addition, the funnel plot (Figure 3) showed that most of the studies were on the top of the inverted-funnel and bilaterally symmetrical which indicate that all the trials were in an unapparent reporting bias.

\section{Heterogeneity analysis}

A total of eight clinical trials were included in this paper. 


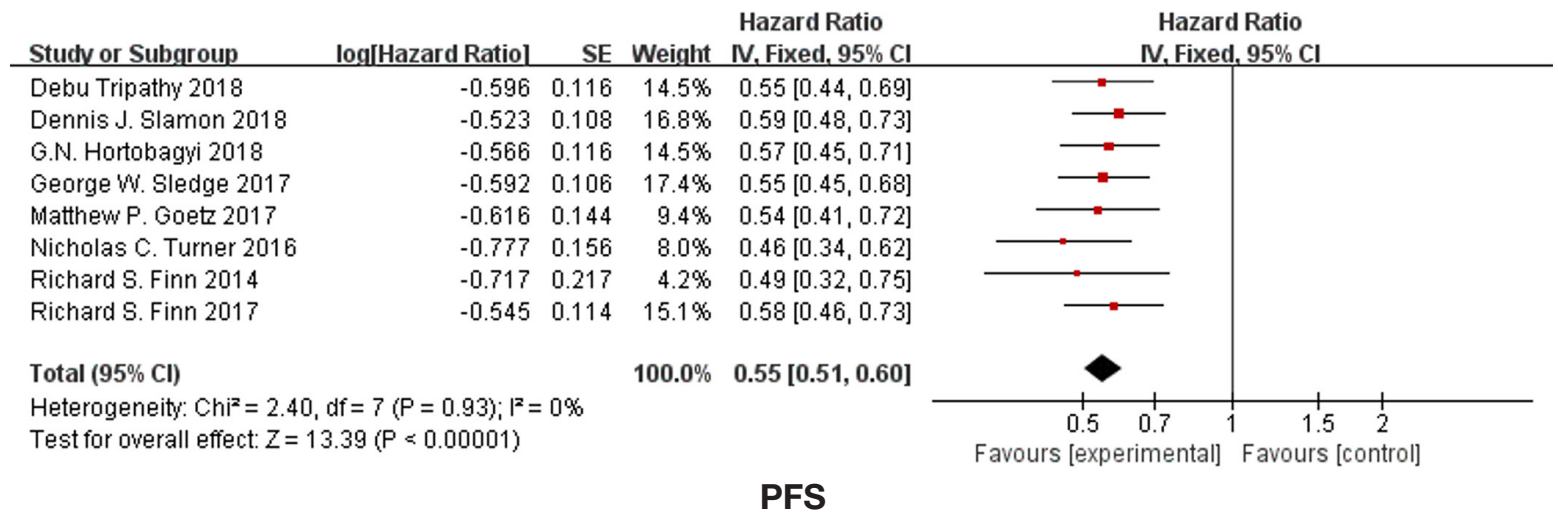

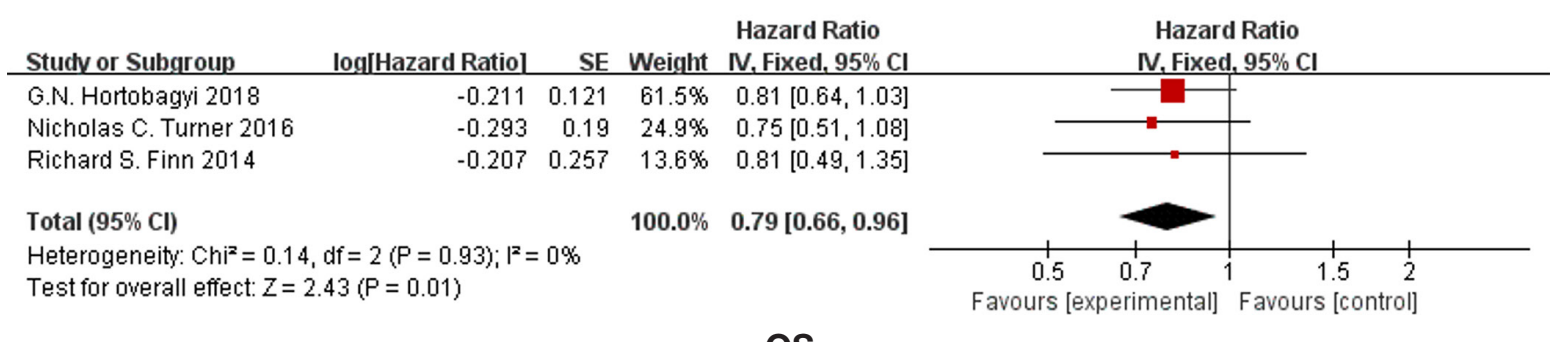

OS

Figure 4 Forest plot of the comparison of efficacy outcome.

The inclusion and exclusion criteria of each clinical trial were similar, and the dose and usage of the same drug in different clinical trials were the same. All trials were random clinical trials, all of which were blind except for Paloma-1, and all of them had the same objectives. It can be concluded that there was little heterogeneity between the included clinical trials, and the results were also verified by statistical heterogeneity in a meta-analysis.

\section{Efficacy assessment}

We used the fixed model to evaluate the pooled PFS because of the low heterogeneity $\left[\mathrm{Chi}^{2}=2.40 \mathrm{df}=7(\mathrm{P}=0.93), \mathrm{I}^{2}=0 \%\right]$. Our results demonstrated that the duration of progressionfree survival (PFS) was significantly longer in the CDK4/6Is group than in the control group (hazard ratio, $0.55 ; 95 \%$ CI, 0.51 to $0.60 ; \mathrm{P}<0.00001$ ) (Figure 4), with the difference between both groups statistically significant. Only three trials provided data for overall survival. Treatment with CDK4/6Is-endocrine therapy resulted in longer overall survival than treatment with endocrine therapy alone (hazard ratio, $0.79 ; 95 \% \mathrm{CI}, 0.66-0.96 ; \mathrm{P}=0.01$ ) (Figure 4) with low heterogeneity $\left[\mathrm{Chi}^{2}=0.14 \mathrm{df}=2(\mathrm{P}=0.93), \mathrm{I}^{2}=0 \%\right]$. The data of OR and CBR were available in all the studies. We used the random model to evaluate the CBR because of the obvious heterogeneity $\left[\mathrm{Chi}^{2}=24.06 \mathrm{df}=7(\mathrm{P}=0.001), \mathrm{I}^{2}=71 \%\right]$, and there was a statistically significant improvement in $\mathrm{RR}$ of CBR with the addition of a CDK4/6Is compared with the endocrine alone (RR, 1.21; 95\% CI, 1.12-1.30; $\mathrm{P}<0.00001)$ (Figure 5). Similar to the CBR, the combination therapy improved OR compared with endocrine therapy alone (RR, 1.48 ; 95\% CI, 1.35 to 1.63 ; $\mathrm{P}<0.00001$ ) (Figure 5) with low heterogeneity $\left[\mathrm{Chi}^{2}=12.26, \mathrm{df}=7(\mathrm{P}=0.09), \mathrm{I}^{2}=43 \%\right]$.

\section{Adverse event}

Eight studies were included in the meta-analysis of adverse events. The main adverse events in each trial were neutropenia, leukopenia, anemia, fatigue, nausea, vomiting and diarrhea (Table 2). According to the outcome of heterogeneity analysis, we used the fixed-effect models to merge the RR of each adverse event in all studies expect neutropenia and diarrhea. We found that the addition of CDK4/6 inhibitors to endocrine therapy could significantly increase the incidence rate of neutropenia, leukopenia, anemia and fatigue (respectively, $\mathrm{RR}=32.04 ; 95 \% \mathrm{CI}$, 17.14-59.90, RR $=30.65 ; 95 \% \mathrm{CI}, 16.51-56.91, \mathrm{RR}=$ 2.82; 95\% CI, 1.85-4.29, RR $=3.92 ; 95 \%$ CI, 2.01-7.68) (Figure 6), and adding CDK4/6Is to endocrine therapy did not increase the incidence rate of gastrointestinal toxicity 


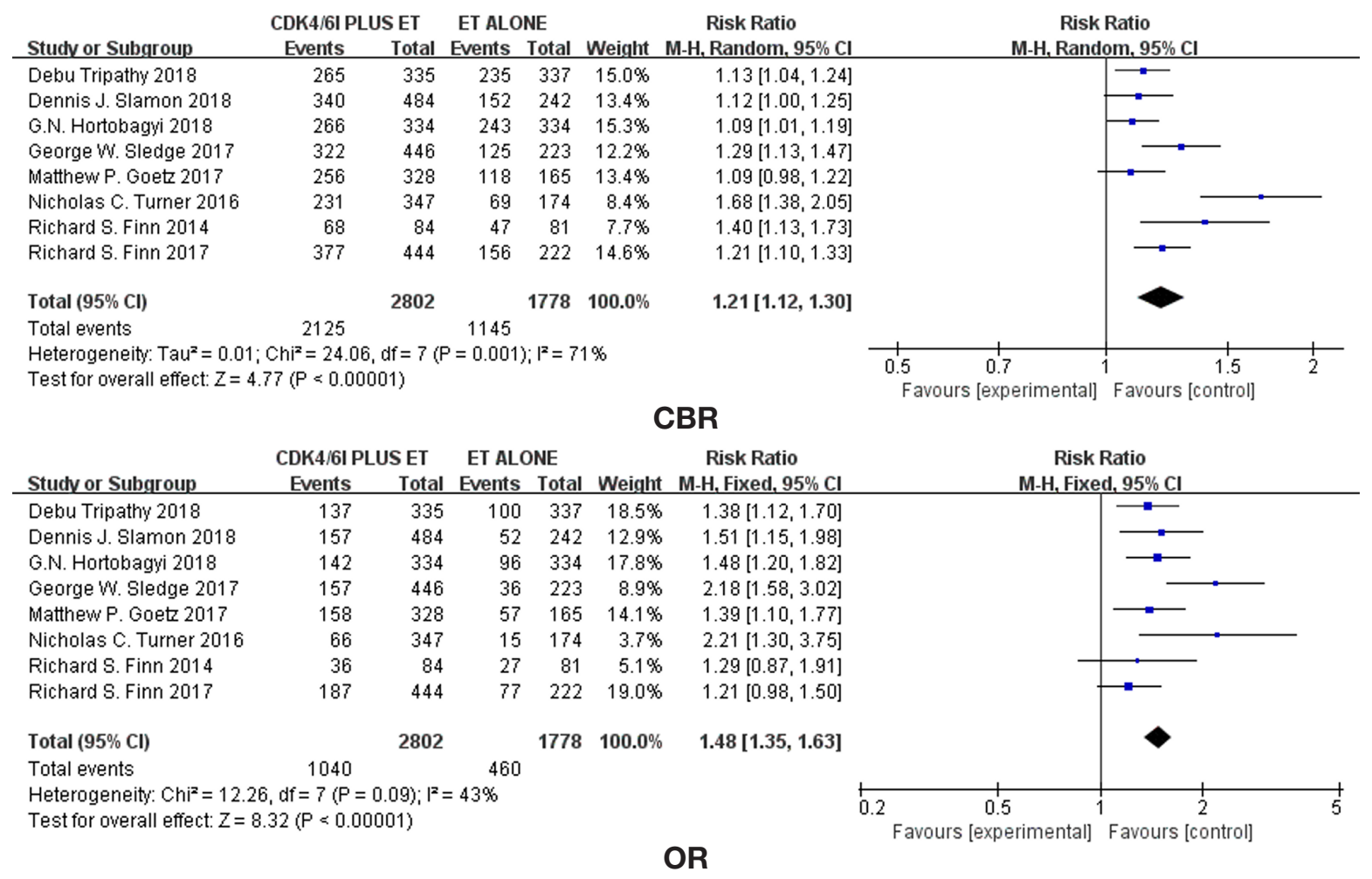

Figure 5 Forest plot of the comparison of efficacy outcome.

Table 2 Treatment-related adverse events (grades III-IV)

\begin{tabular}{|c|c|c|c|c|c|c|c|c|}
\hline Study & Group & $\begin{array}{c}\text { Neutropenia, } \\
\text { n (\%) }\end{array}$ & $\begin{array}{l}\text { Leukopenia, } \\
\text { n (\%) }\end{array}$ & $\begin{array}{l}\text { Anemia, } \\
\text { n (\%) }\end{array}$ & $\begin{array}{c}\text { Diarrhea, } \\
\text { n (\%) }\end{array}$ & $\begin{array}{l}\text { Nausea, } \\
\mathrm{n}(\%)\end{array}$ & $\begin{array}{l}\text { Vomiting, } \\
\text { n (\%) }\end{array}$ & $\begin{array}{c}\text { Fatigue, } \\
\text { n (\%) }\end{array}$ \\
\hline PALOMA-1 & Experimental & $45(54.22)$ & $16(19.28)$ & $5(6.02)$ & $3(3.61)$ & $2(2.41)$ & $0(0)$ & $4(4.82)$ \\
\hline \multirow[t]{2}{*}{ PALOMA-2 } & Experimental & $295(66.44)$ & $110(24.77)$ & $24(5.41)$ & $6(1.35)$ & $1(0.22)$ & $2(0.45)$ & $8(1.80)$ \\
\hline & Control & $3(1.35)$ & $0(0)$ & $4(1.80)$ & $3(1.35)$ & $4(1.80)$ & $3(1.35)$ & $1(0.45)$ \\
\hline PALOMA-3 & Control & $1(0.58)$ & $1(0.58)$ & $3(1.74)$ & $1(0.58)$ & $1(0.58)$ & $1(0.58)$ & $2(1.16)$ \\
\hline \multirow[t]{2}{*}{ MONARCH 2} & Experimental & $117(26.53)$ & $39(8.84)$ & $32(7.26)$ & 59 (13.38) & $12(2.72)$ & $4(0.91)$ & $12(2.72)$ \\
\hline & Control & $4(1.79)$ & $0(0)$ & $2(0.90)$ & $1(0.45)$ & $2(0.90)$ & $4(1.79)$ & $1(0.45)$ \\
\hline MONARCH-3 & Experimental & $69(21.10)$ & $25(7.65)$ & $19(5.81)$ & $31(9.48)$ & $3(0.92)$ & $4(1.22)$ & $6(1.83)$ \\
\hline MONALEESA-2 & Control & $3(0.91)$ & $2(0.61)$ & $4(1.21)$ & $3(0.91)$ & $2(0.60)$ & $3(0.91)$ & $3(0.91)$ \\
\hline \multirow[t]{2}{*}{ MONALEESA-3 } & Experimental & $258(53.42)$ & $68(14.08)$ & $15(3.11)$ & $3(0.62)$ & $7(1.45)$ & $7(1.45)$ & $8(1.66)$ \\
\hline & Control & $0(0)$ & $0(0)$ & $5(2.07)$ & $2(0.83)$ & $2(0.83)$ & $0(0)$ & $1(0.41)$ \\
\hline \multirow[t]{2}{*}{ MONALEESA-7 } & Experimental & $203(60.60)$ & 48 (14.32) & $10(3.00)$ & $5(1.49)$ & $2(0.60)$ & $5(1.49)$ & $4(1.19)$ \\
\hline & Control & $12(3.56)$ & $4(1.19)$ & $7(2.08)$ & $1(0.30)$ & $1(0.30)$ & $2(0.59)$ & $0(0)$ \\
\hline
\end{tabular}




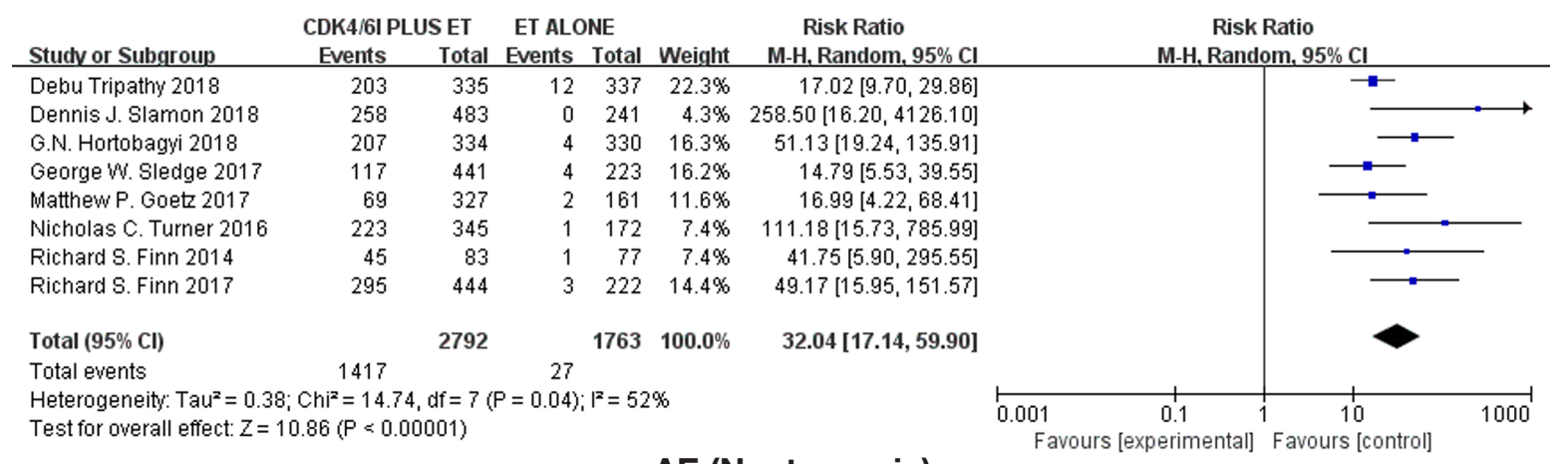

\section{AE (Neutropenia)}

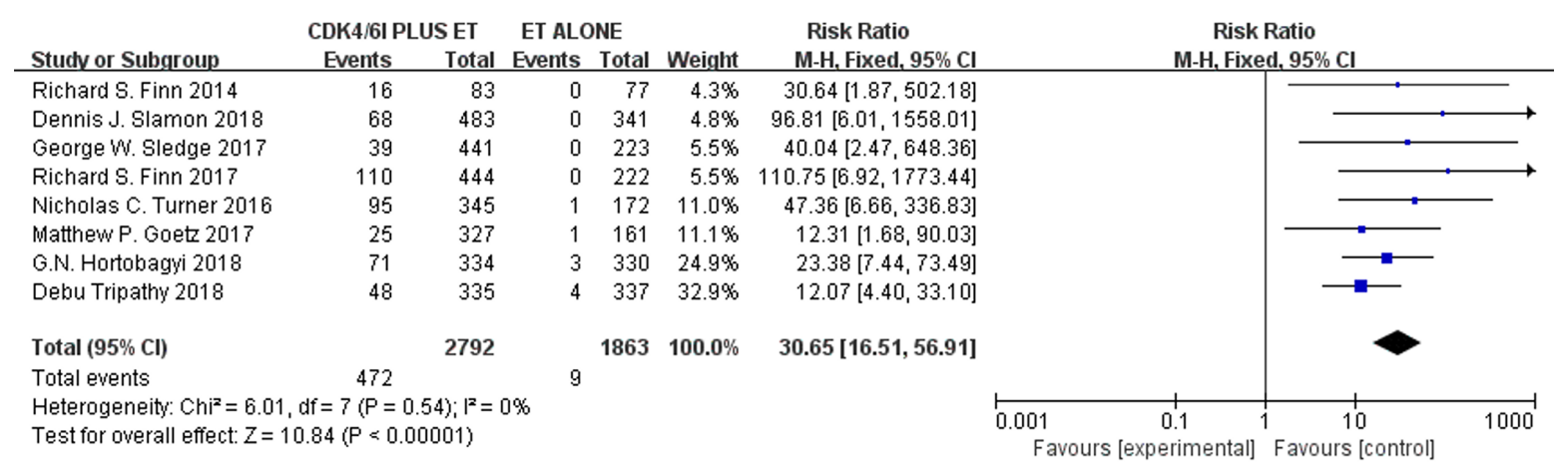

AE (Leukopenia)

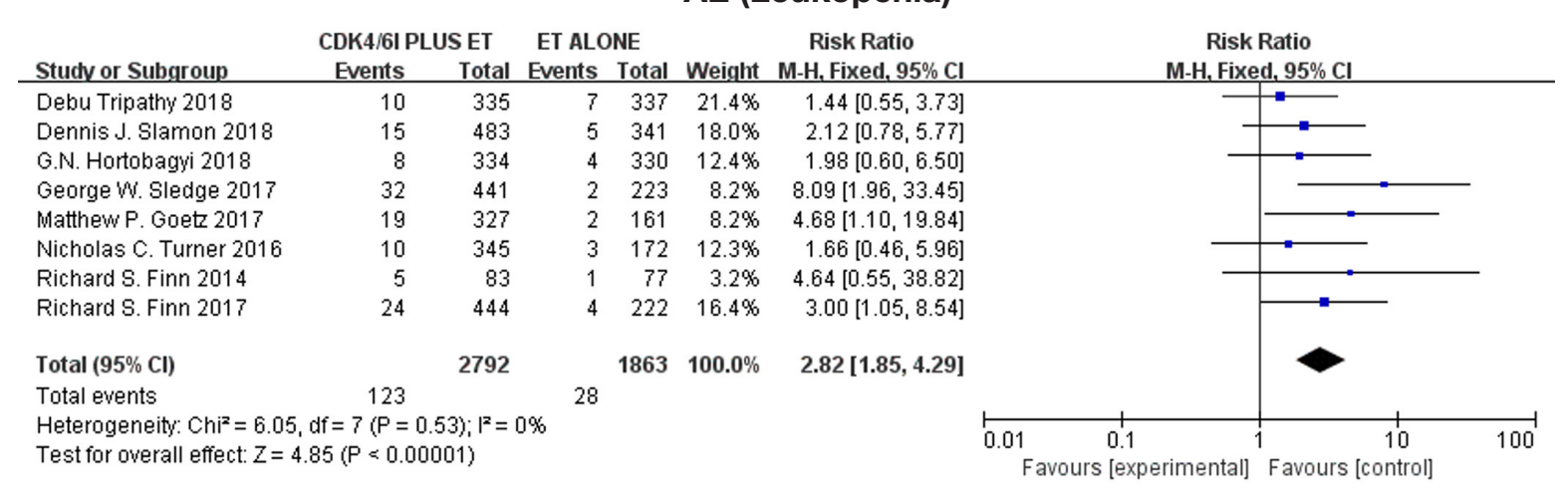

\section{AE (Anemia)}

Figure 6 Forest plot of the comparison of AE (bone marrow suppression). AE, adverse event.

(nausea: $\mathrm{RR}=1.51 ; 95 \% \mathrm{CI}, 0.85-2.68$; diarrhea: $\mathrm{RR}=2.85$; 95\% CI, 1.10-7.42; vomiting: RR =1.31; 95\% CI, $0.74-$ 2.32) (Figure 7).

\section{Discussion}

Breast cancer is the most common cancer posing a serious threat to the health of women worldwide, and advanced breast cancer is still incurable. Therefore, it is important to reduce complications, improve the patient's quality of life and prolong their survival. The treatment of advanced breast cancer includes endocrine therapy, chemotherapy, targeted therapy and so on. A meta-analysis (25) performed by Wilcken indicated that endocrine therapy was better tolerated and had similar OS rates to chemotherapy. The current clinical guideline recommends endocrine therapy as the preferential treatment in HR-positive advanced breast cancer excluding the patients with a rapid progression. Unfortunately, endocrine resistance hampers the survival prolongation of these patients. In recent years, the success 


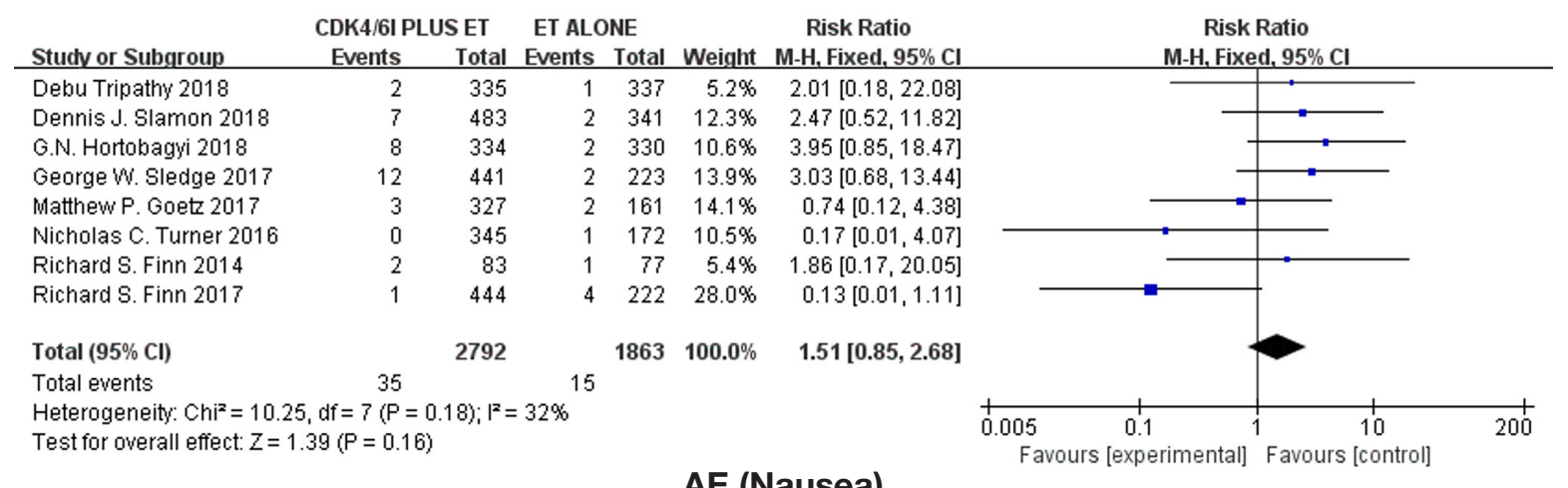

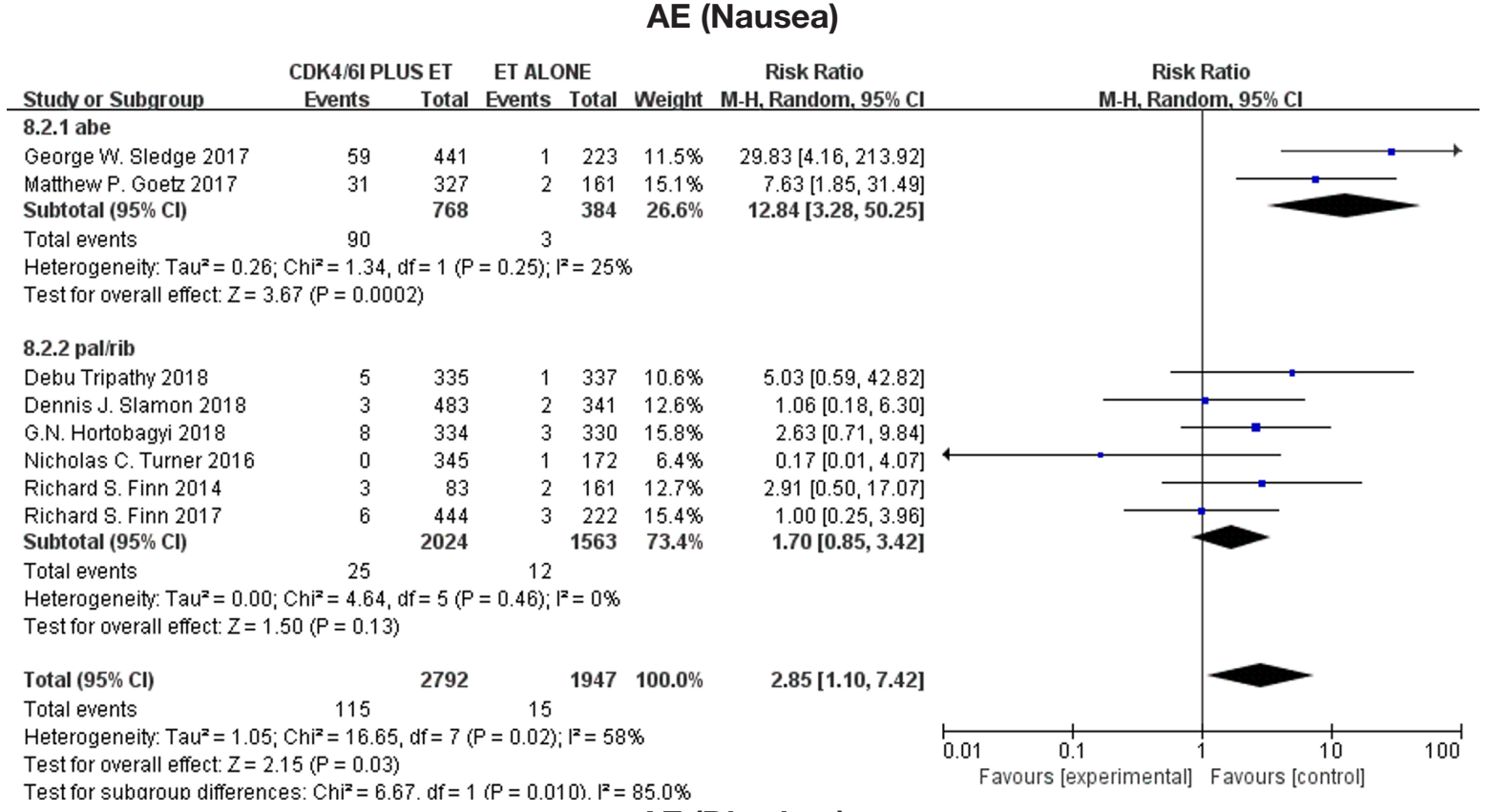

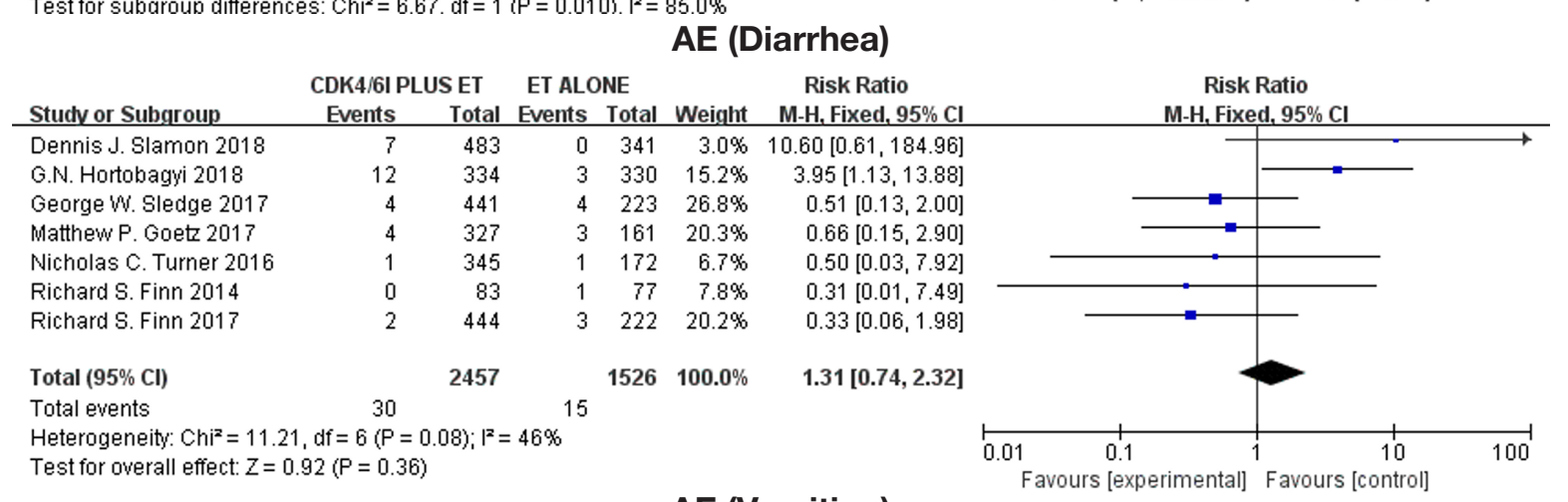

\section{AE (Vomiting)}

Figure 7 Forest plot of the comparison of AE (gastrointestinal toxicity). AE, adverse event.

in the development of highly selective CDK4/6I has brought a new dawn for patients and offers a new approach to the management of advanced breast cancer. To date, the three highly selective CDK4/6Is (palbociclib, ribociclib, and abemaciclib) have been approved by the FDA because of their remarkable clinical curative effect.

The results of this study showed that PFS of the combined treatment regimen was significantly longer than 
that of endocrine monotherapy. In the advanced firstline treatment, the PFS extension time of each clinical trial was about 10 months, indicating that the efficacy of each CDK4/6 inhibitor was positive and relatively stable. At the same time, we conducted subgroup analysis on the treatment timing, drugs, age, race, ECOG score, progesterone receptor status, etc., discovering that the combined treatment regimen had significant benefits in PFS among each subgroup, and there was no heterogeneity in all the pre-specified subgroups except race. The results of subgroup analysis suggest that the Asian population were more sensitive to cdk4/6i than other populations (hazard ratio 0.38 for the Asian versus hazard ratio 0.62 for Non-Asian, $\mathrm{P}$ for difference $=0.002$ ). Only three clinical trials (MONALEESA-2, PALOMA-1, and PALOMA-3) provided OS data. Interestingly, the OS of the combined treatment regimen in each study was slightly longer than that of endocrine monotherapy, but the difference was not statistically significant, while the combined results in this study showed a significant difference. Like the results of PFS and OS, a significant improvement in OR and CBR were also identified in this study. The above results showed that the short-term efficacy of combined therapy in advanced breast cancer could be confirmed, and there was a trend of improvement in OS. Considering the relatively long survival period of breast cancer, a longer follow-up was needed to further evaluate the OS improvement of CDK4/6Is for advanced breast cancer patients. From the above results, we could conclude that the efficacy of the three CDK4/6Is was similar. However, a study (26) showed a more potent ability of abemaciclib to cross the bloodbrain barrier. The data from animal experiments in the study indicated that Abemaciclib brain levels were achieved more efficiently at presumably lower doses than Palbociclib and were most likely on target for a longer period. Besides, research (NCT02302080) has been conducted on the safety and effectiveness of abemaciclib in patients with HRpositive brain metastatic cancer.

Among these clinical trials, bone marrow suppression was the most common grade $3 / 4$ adverse event of patients who received CDK4/6Is, especially leukopenia. This analysis showed a remarkable rise in bone marrow suppression including leukopenia, neutropenia, and anemia. More than $55 \%$ of patients received combined regimen in PALOMA1, PALOMA2, PALOMA3, MONALEESA2, and MONALEESA3 suffered from leukopenia. In contrast, bone marrow suppression in patients who received Abemaciclib appeared to be less common. Grade
$3 / 4$ neutropenia occurring in a combination regimen of MONARCH2 and MONARCH3 were $26.5 \%$ and $21.1 \%$ respectively. Compared with cytotoxic chemotherapymediated cell death, cell-cycle arrest is the major reason for CDK4/6Is-associated bone marrow suppression. Despite the high rate of neutropenia, there was almost no patients who experienced a fever and infrequent clinically relevant infections in all the studies. Furthermore, CDK4/6Isassociated neutropenia can be resolved in 7-14 days after dose interruption, without the drug intervention (27). Gastrointestinal toxicity, such as diarrhea, nausea, vomiting, and abdominal pain, was the other major side effect in these clinical trials. Our study suggested that adding CDK4/6Is to endocrine therapy did not increase the incidence rate of gastrointestinal toxicity with low heterogeneity except diarrhea $\left[\mathrm{Chi}^{2}=16.65, \mathrm{df}=7(\mathrm{P}=0.02) ; \mathrm{I}^{2}=58 \%\right.$, see Figure 7]. According to the characteristics of the data, we carried out subgroup analysis according to the type of drugs and found that patients treated with Abemaciclib seemed to be more prone to suffering from diarrhea $(\mathrm{P}$ for difference $=0.01$, see Figure 7). The chemical structure of Abemaciclib is different from Palbociclib and Ribociclib. Furthermore, the pharmacokinetic properties, especially $\mathrm{IC}_{50}$ values and ratios of CDK4:CDK6 inhibition, are also different in these three highly selective CDK4/6Is. Whether these diversities are relevant to the efficacy and toxicity is unclear and needs further clinical data to confirm.

All patients included in the clinical trials had advanced breast cancer with HR-positive, Her-2 negative. Therefore, we couldn't get sufficient data about the efficacy of CDK4/6Is in different molecular subtype breast cancer and the first-line treatment regimen. In a phase I study performed by Fujiwara (28), one patient with HRnegative and Her-2 positive breast cancer benefitted from Abemaciclib, showing a potential effect of Abemaciclib in Her-2 positive breast cancer. Asghar (29) investigated the sensibility of a different subgroup of triple negative breast cancer patients to CDK4/6Is and finally came to the conclusion that the luminal androgen receptor triple negative breast cancer cell lines were highly sensitive to Palbociclib and Ribociclib. The efficacy of CDK4/6Is in recovery treatment in different molecular subtype breast cancers was supported by many clinical trials. The only preliminary data from four studies (30-33) was about CDK4/6Is used in neoadjuvant therapy. Among them, three clinical trials (30-32) reported that the combination regimen of CDK4/6Is plus aromatase inhibitor could significantly reduce Ki67 expression. Furthermore, a phase 
II neoadjuvant (33) study reported that the overall response rate (ORR) was $89 \%$ and the pathological complete response rate was $11 \%$ in the combination regimen. Although preclinical studies have confirmed the efficacy of CDK4/6Is in some subtype breast cancers $(29,34)$ and in neoadjuvant therapy (30-33), a large number of clinical trials are needed for widespread use.

Reliable and reproducible markers are needed to accurately identify patients who can benefit from these agents. Preclinical studies have shown that an intact, functional $\mathrm{Rb}$ protein plays a prominent role in response to palbociclib (35). Patients with higher levels of $\mathrm{Rb}$ protein were more likely to be more sensitive to CDK4/6Is $(35,36)$. As for other potential biomarkers, a single-agent phase II trial (37) found that advanced breast cancer patients with higher $\mathrm{Rb}$ nuclear expression lower than the Ki67 indices and/or loss of p16 could attain the frequency of response evaluation. A similar result occurred in the analysis from the PALOMA-1/TRIO18 (38). The Ki67 values and the CCND1 expression of ABC patients had a predictive value in the treatment with CDK 4/6Is. However, another biomarker-analysis (39) indicated that the cyclin D1 and Ki67 index values did not influence the clinical outcome. And a biomarker-analyses from PALOMA-2 trial (39) indicated that there were not biomarkers more sensitive than ERpositive to reflect CDK4/6Is response or resistance. In general, there were several candidate biomarkers $(\mathrm{Rb}, \mathrm{p} 16$, $\mathrm{Ki}-67$ index, CCND1 or HR-positive) but they all lack sufficient clinical evidence. More biomarker-analyses studies are needed to determine the optimum biomarker, even the optimum biomarker-combination.

It is necessary to emphasize some deficiencies in this meta-analysis. First, inconsistencies in the follow-up time of each clinical trial may affect the final outcome. Secondly, CDK4/6Is includes three types of drugs, although there is no obvious evidence that the efficacy of the three types of drugs is different, it still affects the result. Finally, most clinical trials are still in progress; they have failed to obtain data on the OS and evaluate the long-term efficacy of drugs in patients.

\section{Conclusions}

It is obvious that high-selective CDK4/6Is is a kind of well-tolerated, effective and oral drug in advanced breast cancer patients with HR-positive and Her-2 negative. Fully understanding the regulation of cell-cycle and signal path can contribute to the use of CDK4/6Is in breast cancer patients and develop an optimum biomarker, even the optimum biomarker-combination. Fully understanding the adverse event of CDK4/6Is is beneficial to the management of these drugs in clinical treatment.

\section{Acknowledgments}

Funding: (I) The National Science Foundation of China (No. 30772587). (II) The Natural Science Foundation of Fujian Province (No. 2011J01188, No. 2016J01769). (III) Fujian Health Project (No. 2011-CXB-8). (IV) Fujian Medical Innovation Project (No. 2018-CX-40).

\section{Footnote}

Conflicts of Interest: All authors have completed the ICMJE uniform disclosure form (available at http://dx.doi. org/10.21037/tcr.2019.11.46). The authors have no conflicts of interest to declare.

Ethical Statement: The authors are accountable for all aspects of the work in ensuring that questions related to the accuracy or integrity of any part of the work are appropriately investigated and resolved.

Open Access Statement: This is an Open Access article distributed in accordance with the Creative Commons Attribution-NonCommercial-NoDerivs 4.0 International License (CC BY-NC-ND 4.0), which permits the noncommercial replication and distribution of the article with the strict proviso that no changes or edits are made and the original work is properly cited (including links to both the formal publication through the relevant DOI and the license). See: https://creativecommons.org/licenses/by-ncnd/4.0/.

\section{References}

1. Global Burden of Disease Cancer Collaboration, Fitzmaurice C, Dicker D, et al. The Global Burden of Cancer 2013. JAMA Oncol 2015;1:505-27.

2. Siegel RL, Miller KD, Jemal A. Cancer statistics, 2016. CA Cancer J Clin 2016;66:7-30.

3. Perou CM, Sorlie T, Eisen MB, et al. Molecular portraits of human breast tumours. Nature 2000;406:747-52.

4. Nadji M, Gomez-Fernandez C, Ganjei-Azar P, et al. Immunohistochemistry of Estrogen and Progesterone Receptors Reconsidered. Am J Clin Pathol 2005;123:21-7. 
5. Shah AN, Cristofanilli M. The Growing Role of CDK4/6 Inhibitors in Treating Hormone Receptor-Positive Advanced Breast Cancer. Curr Treat Options Oncol 2017;18:6.

6. Osborne CK, Schiff R. Mechanisms of endocrine resistance in breast cancer. Annu Rev Med 2011;62:233-47.

7. Thangavel C, Dean JL, Ertel A, et al. Therapeutically activating RB: reestablishing cell cycle control in endocrine therapy-resistant breast cancer. Endocr Relat Cancer 2011;18:333-45.

8. Asghar U, Witkiewicz AK, Turner NC, et al. The history and future of targeting cyclin-dependent kinases in cancer therapy. Nat Rev Drug Discov 2015;14:130-46.

9. Benson C, White J, De Bono J, et al. A phase I trial of the selective oral cyclin-dependent kinase inhibitor seliciclib (CYC202; R-Roscovitine), administered twice daily for 7 days every 21 days. Br J Cancer 2007;96:29-37.

10. Ma CX, Ellis MJ, Petroni GR, et al. A phase II study of $\mathrm{UCN}-01$ in combination with irinotecan in patients with metastatic triple negative breast cancer. Breast Cancer Res Treat 2013;137:483-92.

11. Kortmansky J, Shah MA, Kaubisch A, et al. Phase I trial of the cyclin-dependent kinase inhibitor and protein kinase C inhibitor 7-hydroxystaurosporine in combination with Fluorouracil in patients with advanced solid tumors. J Clin Oncol 2005;23:1875-84.

12. Sausville EA, Arbuck SG, Messmann R, et al. Phase I trial of 72-hour continuous infusion UCN-01 in patients with refractory neoplasms. J Clin Oncol 2001;19:2319-33.

13. Jessen BA, Lee L, Koudriakova T, et al. Peripheral white blood cell toxicity induced by broad spectrum cyclin-dependent kinase inhibitors. J Appl Toxicol 2007;27:133-42.

14. Tan AR, Yang X, Berman A, et al. Phase I trial of the cyclin-dependent kinase inhibitor flavopiridol in combination with docetaxel in patients with metastatic breast cancer. Clin Cancer Res 2004;10:5038-47.

15. Finn RS, Crown JP, Lang I, et al. The cyclin-dependent kinase 4/6 inhibitor palbociclib in combination with letrozole versus letrozole alone as first-line treatment of oestrogen receptor-positive, HER2-negative, advanced breast cancer (PALOMA-1/TRIO-18): a randomised phase 2 study. Lancet Oncol 2015;16:25-35.

16. Ramos-Esquivel A, Hernandez-Steller H, Savard MF, et al. Cyclin-dependent kinase 4/6 inhibitors as firstline treatment for post-menopausal metastatic hormone receptor-positive breast cancer patients: a systematic review and meta-analysis of phase III randomized clinical trials. Breast Cancer 2018;25:479-88.

17. Higgins JP, Green S. Cochrane Handbook for Systematic Reviews of Interventions. Naunyn-Schmiedebergs Archiv für experimentelle Pathologie und Pharmakologie 2011;5:S38.

18. Sledge GW Jr, Toi M, Neven P, et al. MONARCH 2: Abemaciclib in Combination with Fulvestrant in Women with HR+/HER2- Advanced Breast Cancer Who Had Progressed While Receiving Endocrine Therapy. J Clin Oncol 2017;35:2875-84.

19. Goetz MP, Toi M, Campone M, et al. MONARCH 3 : Abemaciclib As Initial Therapy for Advanced Breast Cancer. J Clin Oncol 2017;35:3638-46.

20. Slamon DJ, Neven P, Chia S, et al. Phase III Randomized Study of Ribociclib and Fulvestrant in Hormone ReceptorPositive, Human Epidermal Growth Factor Receptor 2-Negative Advanced Breast Cancer: MONALEESA-3. J Clin Oncol 2018;36:2465-72.

21. Tripathy D, Im SA, Colleoni M, et al. Ribociclib plus endocrine therapy for premenopausal women with hormone-receptor-positive, advanced breast cancer (MONALEESA-7): a randomised phase 3 trial. Lancet Oncol 2018;19:904-15.

22. Hortobagyi GN, Stemmer SM, Burris HA, et al. Updated results from MONALEESA-2, a phase III trial of firstline ribociclib plus letrozole versus placebo plus letrozole in hormone receptor-positive, HER2-negative advanced breast cancer. Ann Oncol 2018;29:1541-7.

23. Cristofanilli M, Turner NC, Bondarenko I, et al. Fulvestrant plus palbociclib versus fulvestrant plus placebo for treatment of hormone-receptor-positive, HER2negative metastatic breast cancer that progressed on previous endocrine therapy (PALOMA-3): final analysis of the multicentre, double-blind, phase 3 randomised controlled trial. Lancet Oncol 2016;17:425-39.

24. Finn RS, Martin M, Rugo HS, et al. Palbociclib and Letrozole in Advanced Breast Cancer. New Engl J Med 2016;375:1925-36.

25. Wilcken N, Hornbuckle J, Ghersi D. Chemotherapy alone versus endocrine therapy alone for metastatic breast cancer. Cochrane Database Syst Rev 2003;(2):CD002747.

26. Raub TJ, Wishart GN, Kulanthaivel P, et al. Brain Exposure of Two Selective Dual CDK4 and CDK6 Inhibitors and the Antitumor Activity of CDK4 and CDK6 Inhibition in Combination with Temozolomide in an Intracranial Glioblastoma Xenograft. Drug Metab Dispos 2015;43:1360-71.

27. Infante JR, Cassier PA, Gerecitano JF, et al. A Phase I 
Study of the Cyclin-Dependent Kinase 4/6 Inhibitor Ribociclib (LEE011) in Patients with Advanced Solid Tumors and Lymphomas. Clin Cancer Res 2016;22:5696-705.

28. Fujiwara Y, Tamura K, Kondo S, et al. Phase 1 study of abemaciclib, an inhibitor of CDK 4 and 6, as a single agent for Japanese patients with advanced cancer. Cancer Chemother Pharmacol 2016;78:281-8.

29. Asghar US, Barr AR, Cutts R, et al. Single-Cell Dynamics Determines Response to CDK4/6 Inhibition in Triple-Negative Breast Cancer. Clin Cancer Res 2017;23:5561-72.

30. Curigliano G, Gomez Pardo P, Meric-Bernstam F, et al. Ribociclib plus letrozole in early breast cancer: A presurgical, window-of-opportunity study. Breast 2016;28:191-8.

31. Ma CX, Gao F, Luo J, et al. NeoPalAna: Neoadjuvant Palbociclib, a Cyclin-Dependent Kinase 4/6 Inhibitor, and Anastrozole for Clinical Stage 2 or 3 Estrogen ReceptorPositive Breast Cancer. Clin Cancer Res 2017;23:4055-65.

32. Hurvitz S, Abad MF, Rostorfer R, et al. Breast cancer, early stage Interim results from neoMONARCH: A neoadjuvant phase II study of abemaciclib in postmenopausal women with $\mathrm{HR}+$ /HER2- breast cancer (BC). Ann Oncol 2016;27S:ESMO \#LBA13.

33. Chow LW, Lam CK, Loo WT. Abstract P6-11-04: OOTR-N007: A phase II neoadjuvant study of letrozole plus palbociclib in postmenopausal patients with ER

Cite this article as: $\mathrm{Xu} \mathrm{ZH}$, Zhang $\mathrm{H}$, Wei DH, Xie LL, Xu CS. Cyclin-dependent kinase 4/6 inhibitor in combination with endocrine therapy versus endocrine therapy only for advanced breast cancer: a systematic review and meta-analysis. Transl Cancer Res 2020;9(2):657-668. doi: 10.21037/tcr.2019.11.46 positive, HER2 negative breast cancer. Cancer Res 2015. doi: 10.1158/1538-7445.SABCS14-P6-11-04.

34. Goel S, Wang Q, Watt AC, et al. Overcoming Therapeutic Resistance in HER2-Positive Breast Cancers with CDK4/6 Inhibitors. Cancer Cell 2016;29:255-69.

35. Dean JL, Thangavel C, McClendon AK, et al. Therapeutic CDK4/6 inhibition in breast cancer: key mechanisms of response and failure. Oncogene 2010;29:4018-32.

36. Finn RS, Dering J, Conklin D, et al. PD 0332991, a selective cyclin D kinase 4/6 inhibitor, preferentially inhibits proliferation of luminal estrogen receptor-positive human breast cancer cell lines in vitro. Breast Cancer Res 2009;11:R77.

37. Clark AS, Lal P, Tan KS, et al. Abstract P2-16-20:

Biomarkers to predict response to the CDK 4/6 inhibitor, palbociclib (PD 0332991) in a single-agent phase II trial in advanced breast cancer. Cancer Res 2013. doi: 10.1158/0008-5472.SABCS13-P2-16-20.

38. Jiang Y, Randolph S, English P, et al. $442 \mathrm{O}$ Cell cycle biomarker analysis from the paloma-1/ trio 18 palbociclib plus letrozole phase ii study in ER-positive/Her2-negative advanced breast cancer (ABC). Ann Oncol 2014;25:iv146.

39. Finn R, Jiang Y, Rugo H. Biomarker analyses from the phase 3 PALOMA-2 trial of palbociclib $(\mathrm{P})$ with letrozole $(\mathrm{L})$ compared with placebo (PLB) plus L in postmenopausal women with ER + /HER2- advanced breast cancer (ABC). Ann Oncol 2016;27:LBA15. 